\title{
DIAGNÓSTICO PRENATAL DE CARDIOPATIA CONGENITA
}

\author{
Erasmo Huertas Tacchino¹, Walter Castillo Urquiaga ${ }^{1}$, Yeimi Suarez Ortega², Silvia Chacón²
}

\section{RESUMEN}

Se presenta el caso de una paciente primigesta sin factores de resgo a la cual se le detecta una cardiopatia congénita tipo CIV muscular la cual es confirmada al nacer mediante ecocardiografía neonatal

Palabras clave: Cardiopatía congénita; Comunicación Interventricular; Ultrasonido(fuente: DeCS BIREME).

\section{PRENATAL DIAGNOSIS OF CONGENITAL HEART}

\begin{abstract}
Presenting the case of a primigravida patient with no risk factors to which it is detected congenital heart disease muscular type VSD is performed which is confirmed by neonatal echocardiography at birth
\end{abstract}

Key words: Congenital heart disease; Septal Comunication; Ultrasound(source: MeSH NLM).

\section{REPORTE DE CASO}

Primigesta de 19 años, nacida en Lima, soltera. sin antecedentes patológicos de importancia; con FUR confiable el 01/01/2013, que inicia su control prenatal a las 11 semanas (22/03) donde le solicitan ecografía, la cual no se realiza hasta el 28/06 en la UMF la cual concluye: Gestacion Unica Activa De 26 Semanas Por biometría fetal, Crecimiento En Percentil 51. Doppler De Uterinas Normales y cardiopatía congénita : CIV Muscular de $3.4 \mathrm{~mm}$ Sin Signos De Insuficiencia Cardiaca, por lo que se sugiere control con ecografía doppler en 5 - 6 semanas (Figura 1). El 29 de Agosto la paciente es evaluada nuevamente en la UMF corroborándose el defecto cardíaco, esta vez con una medida de $3.7 \mathrm{~mm}$ (Figura 2). Finalmente el 27 de setiembre se realiza la tercera y última ecografía donde además del defecto cardíaco se reporta macrosomía fetal (Peso fetal estimado: 4,080 gr) (Figura 3). La

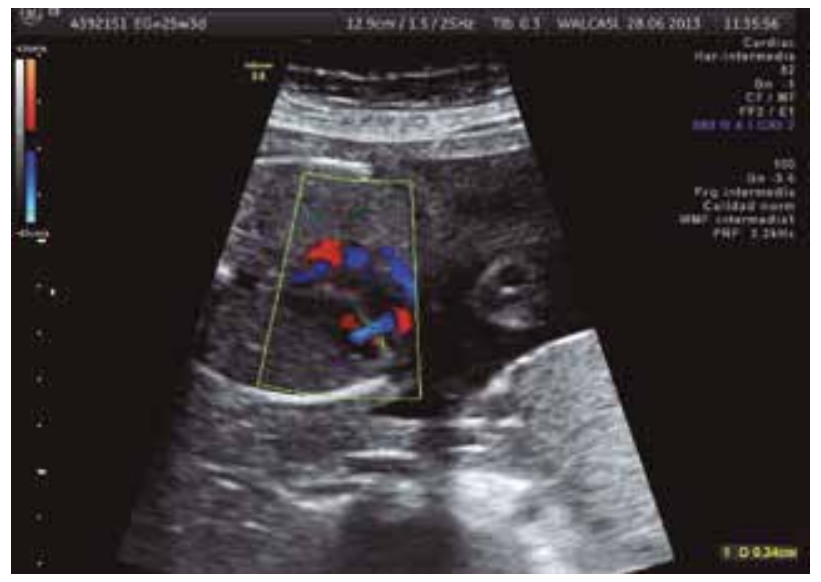

Figura 1. CIV a las 25 semanas.

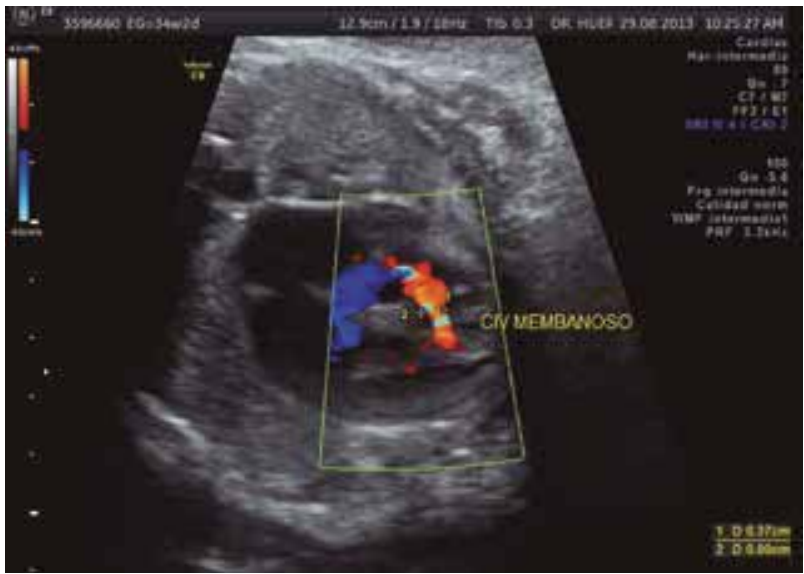

Figura 2. CIV a las 34 semanas.

paciente es vista en consultorios externos donde es programada para cesàrea electiva, la misma que se realiza el 9 de Octubre a las 40 semanas de gestación,

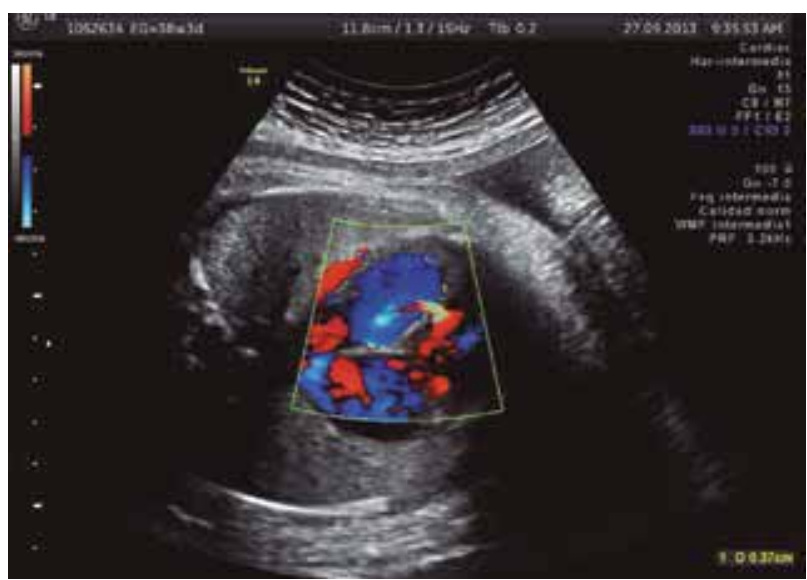

Figura 3. CIV a las 38 semanas.

Médico Ginecólogo Obstetra de la Unidad de Medicina Fetal del Instituto Nacional Materno Perinatal. Lima Perú

2 Médico Residente de Ginecología y Obstetricia del Instituto Nacional Materno Perinatal 
obsteniéndose un RN Mujer de $3918 \mathrm{gr}$ de peso y 51.5 $\mathrm{cm}$ de talla. Apgar 8-9.Liquido Amniotico claro, cantidad normal, no mal olor. Placenta normoinserta fundica. La madre evoluciona favorablemente siendo dada de alta al tercer día. El recien nacido es evaluado en atención inmediata donde se constata un soplo sistólico II/IV. No distress, no cianosis. Evoluciona sin complicaciones por lo que es dado de alta jnto con su madre. El dia 5 de vida es visto por cardiología realizandosele un EKG el cual es informado como normal (Ritmo sinusal. $\mathrm{FC}=$ 153. Eje $+90 . \quad P R=0.20 \mathrm{ST}=\mathrm{N}$. $\mathrm{T}=\mathrm{N}$.) y luego una ecocardiografía la cual concluye: Situs solitus, levocardia. CIV $2 \mathrm{~mm}$ muscular.

\section{DISCUSION}

Las cardiopatías congénitas son las anomalías mayores más frecuentes al nacer, sin embargo el diagnóstico prenatal de las mismas varía entre el 4.5 y $45 \%(1,2)$ debido a factores como la Experiencia del examinador, la Obesidad de la paciente, la Presencia de cicatrices abdominales, la Edad gestacional al momento del examen, la Frecuencia del transductor, el Volumen de líquido amniótico y finalmente la Posición fetal.

La comunicación Interventricular (CIV) es una de las cardiopatías congénitas mas frecuentemente diagnosticadas intrautero con una prevalencia entre 7 y $20 \%(2,3,4)$. Su incidencia sería aún mayor si incluyéramos entre ellas a las CIV que forman parte indisoluble de otras cardiopatías como en la tetralogía Fallot, el síndrome de coartación de la aorta, el tronco arterioso, la doble emergencia del ventrículo derecho y aquellas otras CIV que pueden o no acompañar otras cardiopatías como la transposición de grandes arterias. El pronóstico depende tanto de la localizacion como del tamaño del defecto. En el presente caso por tratarse de un defecto de localización muscular (trabecular) y se pequeño $(2 \mathrm{~mm})$ tiene un buen pronóstco ya que la asociación con aneuploidías es baja y la tasa de cierre espontáneo en el primer año de vida alta. El presente caso demuestra el alto nivel de equipamiento y entrenamiento de los médicos de la unidad de medicina fetal quienes mediante el tamizaje cardiaco sistemático a todas las pacientes que acuden a la ecografía de rutina entre 20 y 24 semanas logran detectar defectos como el presente que podrían pasar desapercibidos.

\section{REFERENCIAS BIBLIOGRÁFICAS}

1. Vignals F., Enriquez G. Detección Prenatal de Cardiopatías Congénitas. REV. MED. CLIN. CONDES - 2008; 19(3) 178 184

2. Garne E., Stoll C., Clementi M., And The Euroscan Group. Evaluation of prenatal diagnosis of congenital heart diseases by ultrasound: experience from 20 European registries. Ultrasound Obstet Gynecol 2001; 17: 386-391

3. Velasquez B, Gallardo J, Acevedo S, Guzman M. Abordaje diagnóstico de la cardiopatía fetal en el Instituto de Perinatología. Ginecol Obstet Mex 2008;76(8):431-9

4. Mayorga C., Rodriguez J, Enriquez G., Alarcon J., Gamboa C., Capella D., Fischer D. Cardiopatías congénitas: diagnóstico prenatal y seguimiento. Rev Chil Obstet Ginecol 2013; 78(5): $349-356$. 